\title{
Acute pancreatitis secondary to cocaine use: a case-based systematic literature review
}

\author{
Muhammad Hassan Naeem Goraya ${ }^{1} \cdot$ Adnan Malik $^{2} \cdot$ Faisal Inayat ${ }^{3}$ (1) Rizwan Ishtiaq $^{4}$. \\ Muhammad Adnan Zaman ${ }^{5} \cdot$ Hafiz Muhammad Arslan ${ }^{3}$ Zahid ljaz Tarar ${ }^{6}$
}

Received: 10 March 2021 / Accepted: 26 April 2021 / Published online: 4 May 2021

(c) Japanese Society of Gastroenterology 2021

\begin{abstract}
Cocaine use continues to be an important global public health problem. As the use of cocaine remains pervasive so have a myriad of adverse events associated with this drug. These deleterious effects are well-studied, but gastrointestinal complications remain esoteric and the existing clinical evidence is scarce. Ischemia of the esophagus and small bowel, perforation, peptic ulceration, gastrointestinal bleeding, and ischemic colitis are among the reported complications. In specific, acute pancreatitis secondary to cocaine use is an exceedingly rare clinicopathologic entity. To date, only 7 cases of this condition have been reported in the English-language literature. We hereby delineate a rare case of a 29 -year-old female who developed her first episode of cocaine-associated pancreatitis. The diagnosis was made based on a standard battery of investigations and meticulous exclusion of common etiologies of acute pancreatitis. To our knowledge, this case represents the first report of re-occurrence of acute pancreatitis upon subsequent crack cocaine insufflation, adding a higher level of evidence to a fallible association. We also present a systematic review of the existing literature on acute pancreatitis following cocaine use. An updated knowledge regarding this rare association is of paramount importance for early diagnosis and astute management.
\end{abstract}

Keywords Acute pancreatitis $\cdot$ Cocaine $\cdot$ Gastrointestinal complications $\cdot$ Rare association $\cdot$ Re-occurrence of pancreatitis

\section{Introduction}

Cocaine is a powerful drug of abuse that has now been increasingly used worldwide. It affects multiple body systems and may result in a wide range of complications [1, 2]. Gastrointestinal adverse effects of cocaine consumption remain relatively uncommon. Published medical literature contains anecdotal reports of prepyloric and duodenal perforations, peptic ulcer disease, gastrointestinal hemorrhage,

Faisal Inayat

faisalinayat@hotmail.com

1 Services Institute of Medical Sciences, Lahore, Punjab, Pakistan

2 Loyola University Medical Center, Maywood, IL, USA

3 Allama Iqbal Medical College, Allama Shabbir Ahmad Usmani Road, Faisal Town, Lahore 54550, Punjab, Pakistan

4 Mercy Health St. Vincent Medical Center, Toledo, OH, USA

5 Wexham Park Hospital, Slough, UK

6 University of Missouri School of Medicine, Columbia, MO, USA bowel ischemia, bowel infarction, ischemic colitis, retroperitoneal fibrosis, and sphincter of Oddi dysfunction [3-6]. However, cocaine-associated acute pancreatitis is an extremely rare entity. To our research, only 7 such cases have been reported in the English-language literature thus far [7-13]. Clinical features, serum levels of pancreatic enzymes, and consistent radiological findings, in combination with exclusion of common causes of pancreatitis are pivotal for establishing the diagnosis [14]. Prompt treatment with adequate fluid resuscitation and pain control usually results in recovery. However, patients may also develop an unpredictable and potentially lethal disease, culminating in systemic complications requiring intensive care unit admission [14]. It is concerning that the underlying etiology of acute pancreatitis remains unknown in approximately $30 \%$ of cases even after extensive diagnostic workup [14]. Therefore, an updated knowledge of under-recognized etiologies of pancreatitis is crucial to diagnose and classify these patients $[15,16]$.

As per the United States Dawn Abuse Warning Network (DAWN) data, cocaine had the highest rates of emergency department visits compared to other illicit drugs (162.1 per 
100,000 population), with a total of 505,224 visits (40.3\%) for the year 2011 [17]. Acute pancreatitis is one of the leading causes of hospital admission in the United States, with approximately 300,000 annual emergency room visits [18]. Pertinently, the global use of cocaine continues to soar in recent times, but pancreatitis following this drug is not commonly reported. Therefore, a heightened awareness of cocaine-related acute pancreatitis is warranted. Further work should be undertaken to figure out this association and risk factors. Prompt drug screening should be mandated in patients under the age of 30 years presenting with symptoms of pancreatitis. In this article, we chronicle the first case of re-occurrence of cocaine-associated acute pancreatitis involving a young female patient on re-exposure to cocaine. In addition, we systematically review the limited number of available studies on this topic.

\section{Illustrative case}

\section{Presentation}

A 29-year-old previously healthy Caucasian female presented to the emergency department with severe abdominal pain radiating to the back for 1 day. The pain was sudden-onset, dull in nature, and worsened gradually. It was associated with nausea and vomiting. She denied diarrhea, melena, hematochezia, constipation, fever, chills, stabbing chest pain, cough, or shortness of breath. The patient was a former 6-pack-year smoker but denied alcoholism. She had been snorting crack cocaine daily for the past several months but denied intravenous drug use. She admitted the last use 2 days prior to her current presentation. She was not on any home medications or herbal supplements. Her past medical and surgical histories were unremarkable. Family history was negative for cancer and familial hypertriglyceridemia. At presentation, her vital sign examination revealed temperature $98.7^{\circ} \mathrm{F}$, blood pressure $102 / 74 \mathrm{mmHg}$, respiratory rate 24 breaths/min, and heart rate 106 beats/min. Physical examination was remarkable for marked tenderness in the epigastrium and right upper quadrant, with hypoactive bowel sounds. No rebound tenderness or abdominal wall rigidity was noted.

\section{Diagnostic workup}

Laboratory studies revealed serum lipase 3586 IU/L, amylase $1267 \mathrm{IU} / \mathrm{L}$, total bilirubin $2.4 \mathrm{mg} / \mathrm{dL}$, alanine aminotransferase $39 \mathrm{IU} / \mathrm{L}$, aspartate aminotransferase $43 \mathrm{IU} / \mathrm{L}$, alkaline phosphatase $124 \mathrm{IU} / \mathrm{L}$, white cell count 10,300/ $\mathrm{uL}$, triglycerides $134 \mathrm{mg} / \mathrm{dL}$, and calcium $9.8 \mathrm{mg} / \mathrm{dL}$. The IgG4 level $(23 \mathrm{mg} / \mathrm{dL})$ was within normal limits. Urine toxicology screen came back positive for cocaine. Her electrocardiogram and chest radiograph were unremarkable for abnormalities. Urinalysis ruled out infection and urine human chorionic gonadotropin tested negative for pregnancy. Coronavirus testing via nasopharyngeal swab was negative. Abdominal ultrasonography revealed a heterogeneous hypoechoic pancreas, normal-sized common bile duct, and a normal gallbladder with no gallstones. Computed tomography scan of the abdomen showed an edematous, heterogeneous pancreas with a subtle non-enhancement in the body region along with significant surrounding fat arborization, consistent with acute pancreatitis (Fig. 1). The patient then underwent a magnetic resonance cholangiopancreatography, which ruled out pancreatic mass. After exclusion of common etiologies of acute pancreatitis, cocaine-induced acute pancreatitis was considered the probable diagnosis.

\section{Treatment and clinical outcome}

The patient was initiated on goal-directed intravenous hydration, oral antiemetics, and morphine. She was administered intravenous 1-L Ringer's lactate bolus on admission and $200 \mathrm{~mL} / \mathrm{h}$ thereafter. She started responding to conservative management with the gradual diminution of clinical symptoms. The trend in her serum lipase and amylase levels also showed a gradual decline toward normal (Fig. 2). The maintenance fluids were decreased and oral intake was initiated on hospital day 4 . She started tolerating a solid diet after 5 days of admission. Optimal pain control was achieved with oral morphine and she did not experience another episode of vomiting. She was discharged from the

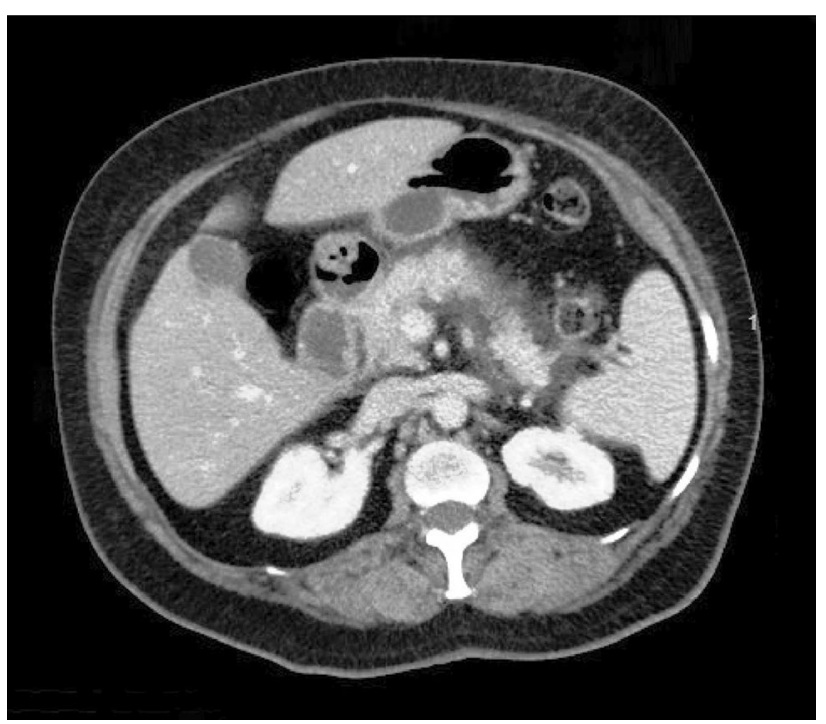

Fig. 1 Contrast-enhanced computed tomography abdomen showing an edematous, heterogenous pancreas with a subtle non-enhancement in the body region along with significant surrounding fat stranding, consistent with acute pancreatitis 
Fig. 2 Graphical representation of the changes in serum lipase and amylase levels during the hospital course of the patient

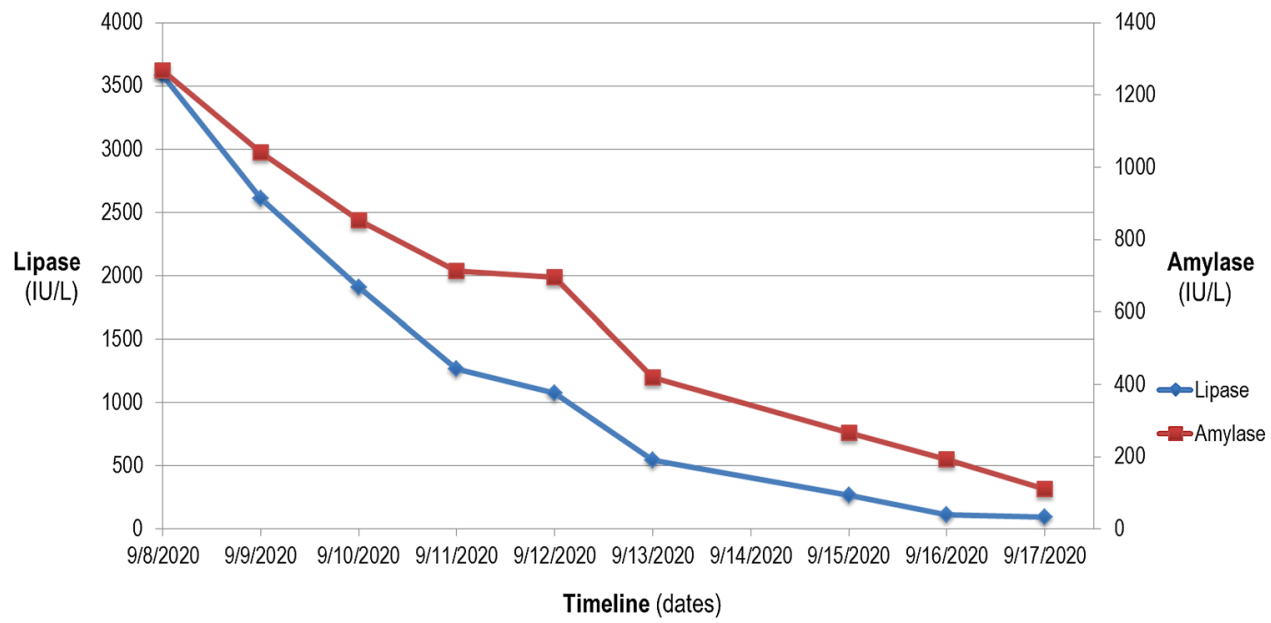

hospital on day 10 of admission in a stable clinical condition with a normal biochemical profile. However, after 2 months, she experienced another episode of severe acute pancreatitis (serum lipase: $9841 \mathrm{IU} / \mathrm{L}$ ) following insufflation of a large amount of crack cocaine. It was successfully managed with conservative treatment. The Naranjo Nomogram for Adverse Drug Reaction Assessment (Naranjo score) was calculated, which resulted in 10 (Table 1). The re-occurrence confirmed the definitive diagnosis of acute pancreatitis following cocaine use in this patient. She was again educated about the etiology of her acute pancreatitis and was directed to seek professional help for substance use disorder. Since then, she has been receiving cognitive-behavioral therapy sessions with remarkable adherence. Her regular urine toxicological screens confirmed abstinence from cocaine and she did not develop another episode of acute pancreatitis to date.

\section{Methods}

A systematic review was performed using the Preferred Reporting Items for Systematic Reviews and Meta-Analyses (PRISMA) guidelines [19]. We queried research databases, including MEDLINE (PubMed and Ovid), Embase, Scopus, and Cochrane for English-only articles published between inception and March 05, 2021. A comprehensive search strategy was constructed using specific terminologies. The search terms, including "cocaine", "coke", "crack", "blow", "snow", "nose candy", "cocaine-related side effects", "complications", and "adverse reactions" were combined using the Boolean operators 'AND' and 'OR' with the terms 'pancreatitis" and "acute pancreatitis" with all associated permutations. The studies based on human subjects only were considered for analysis.

Table 1 Naranjo assessment scale depicting a score of 10 in the present case; a score of $<1$ is doubtful, $1-4$ possible, 5-8 probable, and $>9$ definitive for adverse drug reaction

Naranjo adverse drug reaction probability scale

\begin{tabular}{|c|c|c|c|c|}
\hline Questions & Yes & No & Do not know & Patient's score \\
\hline 1. Are there previous conclusive reports on this reaction? & +1 & 0 & 0 & +1 \\
\hline 2. Did the adverse event appear after the suspected drug was administered? & +2 & -1 & 0 & +2 \\
\hline $\begin{array}{l}\text { 3. Did the adverse reaction improve when the drug was discontinued or a specific antagonist was } \\
\text { administered? }\end{array}$ & +1 & 0 & 0 & +1 \\
\hline 4. Did the adverse event reappear when the drug was re-administered? & +2 & -1 & 0 & +2 \\
\hline 5. Are there alternative causes (other than the drug) that could on their own have caused the reaction? & -1 & +2 & 0 & +2 \\
\hline 6. Did the reaction reappear when a placebo was given? & -1 & +1 & 0 & \\
\hline 7. Was the drug detected in blood (or other fluids) in concentrators known to be toxic? & +1 & 0 & 0 & +1 \\
\hline $\begin{array}{l}\text { 8. Was the reaction more severe when the dose was increased or less severe when the dose was } \\
\text { decreased? }\end{array}$ & +1 & 0 & 0 & +1 \\
\hline 9. Did the patient have a similar reaction to the same or similar drugs in any previous exposure? & +1 & 0 & 0 & \\
\hline 10. Was the adverse event confirmed by any objective evidence? & +1 & 0 & 0 & \\
\hline Total score & & & & 10 \\
\hline
\end{tabular}




\section{Inclusion criteria}

Acute pancreatitis was characterized by fulfilling 2 of the 3 Revised Atlanta Classification criteria: (1) abdominal pain consistent with acute pancreatitis; (2) elevated serum lipase or amylase to more than 3 times the upper range of normal levels; and (3) radiological investigations showing features of acute pancreatitis [20]. Cocaine use was defined in each study by patient report, physician report, or confirmation in urine toxicology screen. Cocaine use parameters were delineated in the most detailed amount provided in each included paper. Cocaine-related acute pancreatitis was designated by the preceding use of cocaine and subsequent development of acute pancreatitis after exclusion of all probable etiologies of pancreatitis, including alcoholism, biliary causes, hypertriglyceridemia, drug-induced disease, autoimmune, and hereditary type of pancreatitis [20-23].

Two of the authors identified potentially relevant studies by independently reviewing the titles and abstracts of each article included in the search results. The database search yielded 217 results (PubMed and Ovid 83, Scopus 75, Embase 50, and Cochrane 9), which were selected for inclusion in the qualitative analysis. Furthermore, we reviewed conference papers from major gastroenterology scientific meetings, including Digestive Disease Week, United European Gastroenterology Week, and American College of Gastroenterology. A manual search of bibliography lists was also undertaken for additional articles. To determine eligibility, full-text versions of potentially relevant articles were independently retrieved and reviewed by the other 2 authors. Disagreements pertaining to eligibility were resolved after joint re-assessment and consensus. The final inclusion of a study into our analysis was based on a discussion headed by the senior author. A vast majority of studies were excluded as they reported other causes of pancreatitis, were redundant or duplicate, did not include human subjects, or the language was other than English. Seven articles met inclusion criteria for cocaine-associated acute pancreatitis. PRISMA flow diagram outlines the search methodology for data synthesis and results (Fig. 3). Two of the authors independently extracted data into a standardized form and validated for agreement. The data on patient demographics, clinical characteristics, time to develop pancreatitis after cocaine abuse, comorbid conditions, laboratory evaluation, abdominal imaging findings, treatment, clinical outcomes, and duration of hospital stay were collected and summarized.

\section{Results}

Acute pancreatitis secondary to cocaine use has been described in a total of 7 case reports only (level of clinical evidence: IV), dating from 2005 to 2020 [7-13]. Cocaineinduced pancreatitis showed a clear male predominance, as all 7 patients were male. The age of patients ranged from 18 to 61 years (mean \pm standard deviation, $45.14 \pm 22$ years). Notably, 5 (71\%) of 7 patients were under 30 years of age. This observation may facilitate prompt suspicion of cocainerelated acute pancreatitis in young cocaine users presenting with acute gastrointestinal symptoms. The race of patients could be an important information but it was not reported in $6(86 \%)$ of 7 previously described cases. In terms of the
Fig. 3 Preferred Reporting Items for Systematic Reviews and Meta-Analyses (PRISMA) flow diagram showing the search methodology for data synthesis regarding cocaineinduced acute pancreatitis

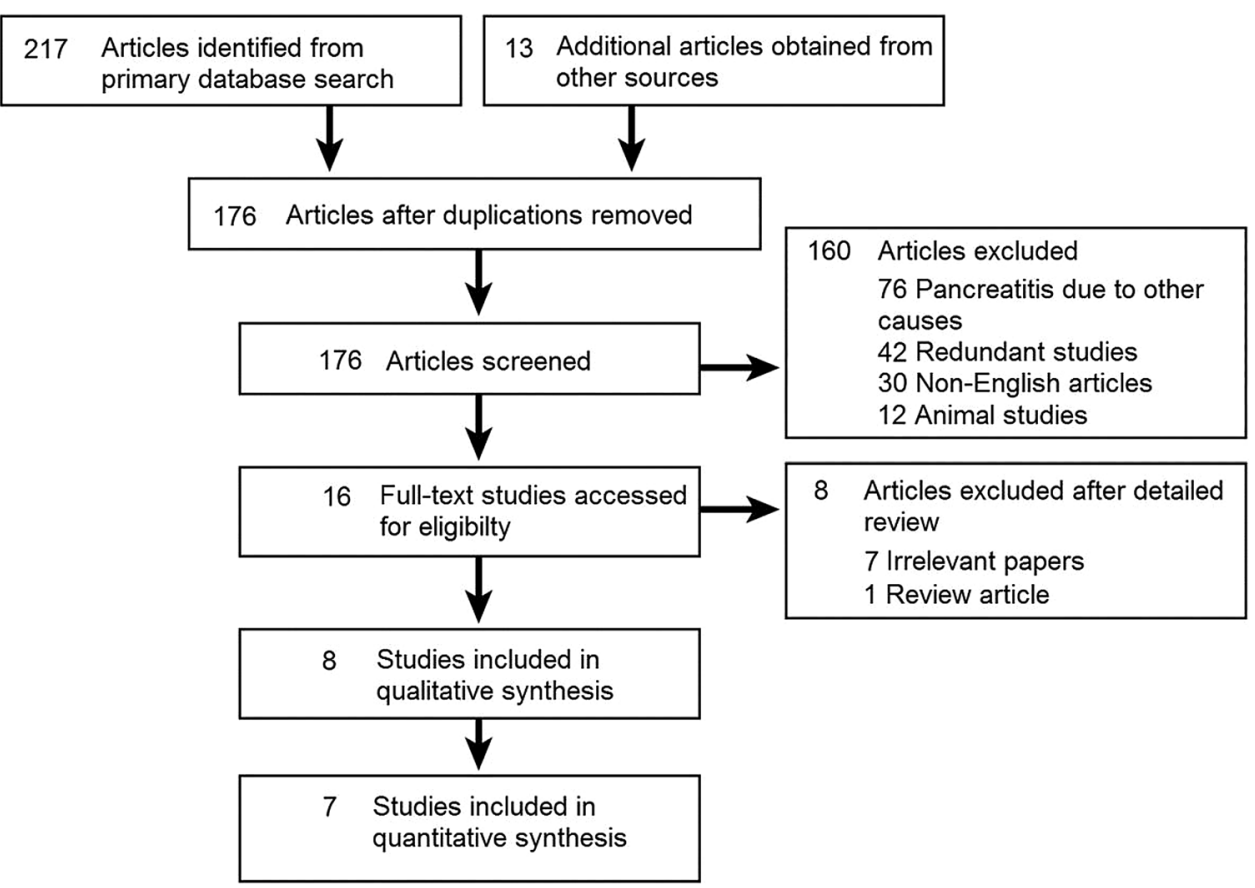


temporal relationship between the disease presentation and cocaine abuse, $6(86 \%)$ of 7 patients developed symptoms within $48 \mathrm{~h}$ of cocaine exposure. Several patients were previously healthy but a few had concurrent chronic medical conditions that were mostly unrelated to the potential risk factors for pancreatitis. Concurrent liver dysfunction may also occur in these patients but it may resolve spontaneously after pancreatitis treatment. In abdominal imaging findings, pancreatitis spread throughout the pancreas in $6(86 \%)$ of 7 patients, whereas $1(14 \%)$ patient had only an increased density of the peripancreatic fat (Table 2).

Polysubstance use disorder was reported in $4(57 \%)$ of 7 , whereas cocaine abuse alone was described in $3(43 \%)$ patients. Based on the radiological investigations, biliary etiologies were ruled out in all 7 patients included in the study. Three patients had a history of alcohol use, but there was no history of intake preceding the acute pancreatitis episode. Genetic studies for a source of acute pancreatitis were not available. Family history was unremarkable in all 7 patients. Hypertriglyceridemia was excluded in all 7 patients, whereas hypercalcemia was excluded in the $3(43 \%)$ patients for whom data were reported. None of the patients had a history of trauma or an overt evidence of infection. Drug-induced acute pancreatitis was ruled out in all 7 patients.

\section{Discussion}

A rare case of acute pancreatitis secondary to cocaine use has been encountered in a young female patient with clinical evidence of re-occurrence upon cocaine re-use and a systematic review of existing data has been presented to raise awareness regarding this unique association that could be increasingly recognized in the clinical practice.

Cocaine is a common drug of abuse that can cause various debilitating complications. Gastrointestinal adverse effects of cocaine are relatively uncommon, with acute pancreatitis being even rarer. Bernad et al. first recognized cocaine-associated acute pancreatitis in 1990 [24]. Since then, this drug has rarely been implicated as an underlying etiology for acute pancreatitis. With regard to pathogenesis, the exact mechanism remains unknown. It is notable that cocaine inhibits the reuptake of norepinephrine and dopamine within the presynaptic nerve endings. It results in increased norepinephrine presence in the synaptic cleft, leading to postsynaptic stimulation. In the gastrointestinal tract, it also causes direct vasoconstriction and thrombosis of mesenteric vessels, which is mediated by the calcium flux across the endothelial cell membrane [11]. It is postulated that the pathophysiological changes causing cocaine-induced pancreatitis involve mechanisms such as vasoconstriction and thrombotic microangiopathy $[7,11]$. Furthermore, levamisole is a common contaminant of cocaine that can cause vasculitis and agranulocytosis due to its immunomodulatory properties [25]. It can be speculated that levamisole may have contributed to the disease presentation in this patient. The pancreatic perfusion was reduced following cocaine use, leading to ischemic changes resulting in the inflammatory response in the form of acute pancreatitis [26, 27].

Permanent cessation of cocaine is imperative in terms of treatment. Conservative management, using adequate pain control, goal-directed intravenous fluid resuscitation, and correction of electrolyte and metabolic problems, is instrumental in the recovery of most patients in 3-7 days $[8,11$, 12]. In addition to acute pancreatitis, patients may also rarely develop concurrent gastric ulceration and bleeding, requiring prompt hemostasis [9]. In severe cases of cocaine-related acute pancreatitis, intensive care unit admission might be required due to associated complications and organ failure $[9,10,12]$. The reported systemic complications include incomplete thrombotic microangiopathy, vasculitis, pauciimmune necrotizing glomerulonephritis, and multiorgan involvement [9, 10, 12]. Extensive management is warranted depending on the complications in such patients. Intravenous prednisone, plasmapheresis, and hemodialysis have been employed in this regard $[9,10,12]$. Enteral nutrition using a nasojejunal tube can also be administered in severe cases [28]. Supplemental parenteral nutrition is used if the required rate is not achieved in up to $72 \mathrm{~h}$ and pancreatitis remains unresolved [28].

The current patient was diagnosed with cocaineinduced acute pancreatitis after extensive diagnostic workup and exclusion of probable etiologies. Her medical history was negative for alcohol abuse. Abdominal ultrasound ruled out the gallstones and other abnormalities in the gallbladder. Furthermore, her serum triglyceride levels were within normal limits and her urine pregnancy test was negative [29]. She had normal serum IgG4 levels, no history of prior pancreatitis episodes, and no evidence of IgG4-associated autoimmune systemic disease or concomitant inflammatory bowel disease. According to the International Consensus Diagnostic Criteria, a probable diagnosis of autoimmune pancreatitis was excluded [30, 31]. For precise etiology establishment, it is imperative to differentiate between drug-induced disease and acute pancreatitis following cocaine use. Therefore, clinicians should maintain an updated knowledge of the potential drug associations of acute pancreatitis $[32,33]$. The clinical history-taking, focusing the details of underlying medical conditions, plays a key role in this regard. The patient involved in this study had no underlying chronic illnesses. She was not on any medications or herbal supplements before or at the time of admission. She admitted crack cocaine insufflation and her urine drug screen was also positive for cocaine only. The close temporal relationship between cocaine use and symptom onset followed by 


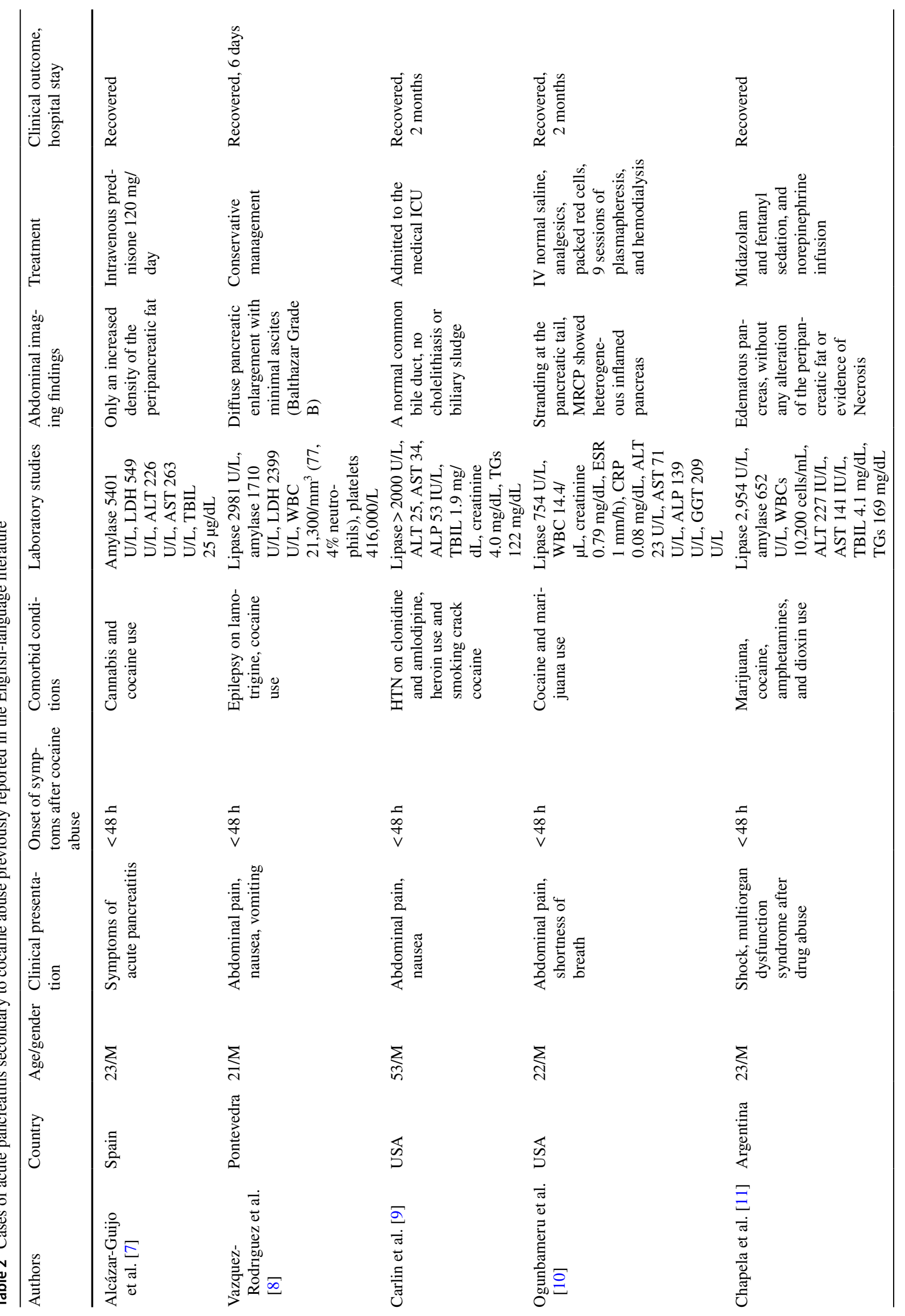




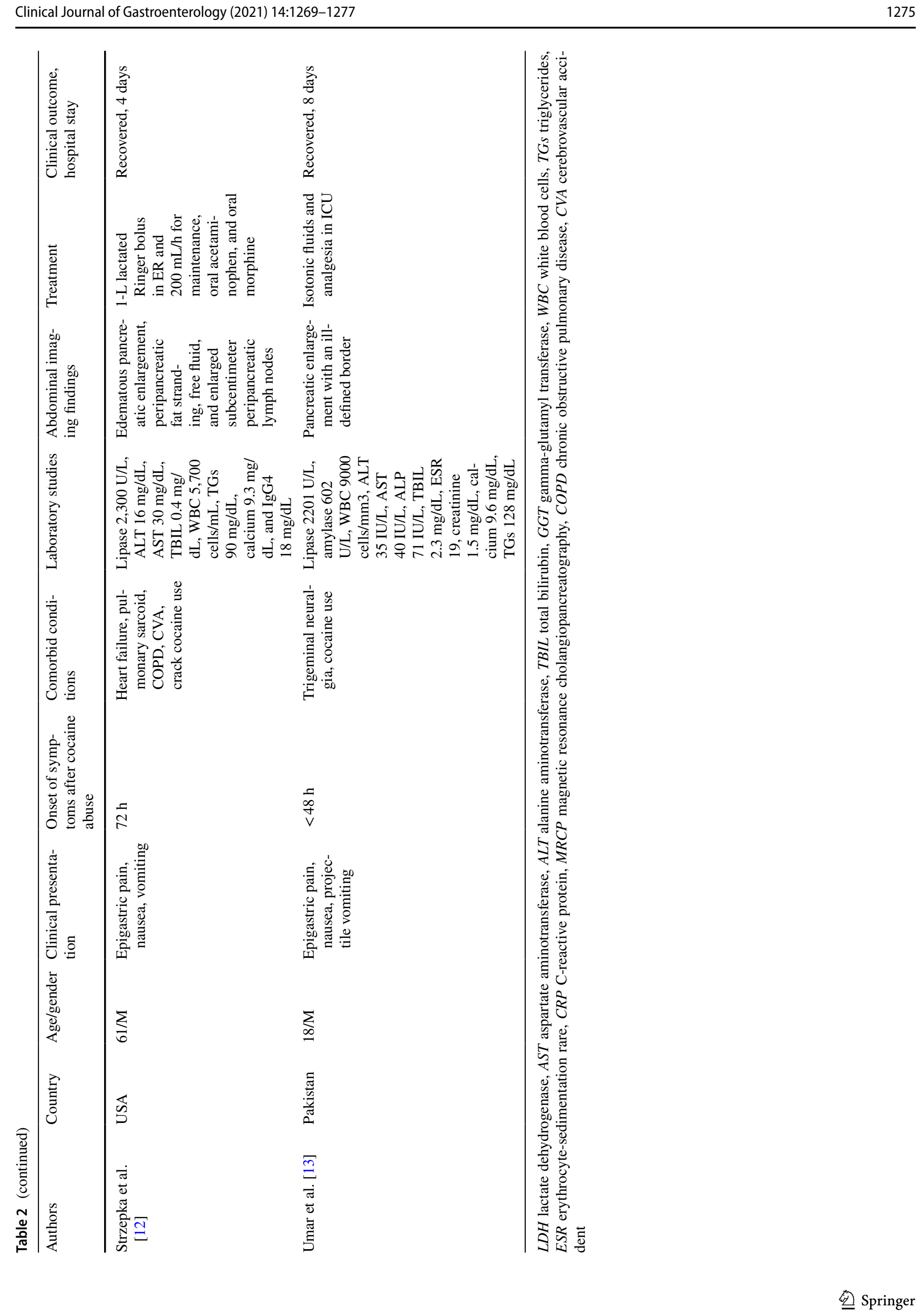


clinical and biochemical improvement after conservative management supplemented the notion that the etiology behind her first episode of acute pancreatitis was cocaine.

The Naranjo algorithm is a frequently used causality assessment tool that can be indicative of an appropriate exclusion of alternative etiologies in cases of acute pancreatitis [34]. In the present case, the following Naranjo scale findings turned out to be positive: prior conclusive reports exist regarding this reaction, the acute pancreatitis occurred after cocaine use, the adverse reaction improved after the drug was discontinued, the patient experienced re-occurrence upon re-use of cocaine after 2 months, alternative causes of acute pancreatitis were carefully excluded, cocaine was detected in urine of the patient, and the second episode of pancreatitis was more severe as she had a large amount of crack cocaine. It resulted in a Naranjo score of 10 , which indicates acute pancreatitis as a definitive adverse drug reaction in this case. The existing literature on this association is primarily based on a few case reports, which makes it tenuous. In previously described cases, information regarding re-occurrence of cocaineassociated acute pancreatitis is not available. Therefore, to the best of our knowledge, this is the first report of acute pancreatitis detailing re-occurrence of disease upon a cocaine relapse. It is a novel finding as re-occurrence denotes confirmation of the association between cocaine use and acute pancreatitis.

In conclusion, cocaine may be added to the list of plausible causes of acute pancreatitis, especially in cocaine users presenting with abdominal pain. Clinicians should be cognizant of this etiology as it can potentially help to explain some cases of idiopathic acute pancreatitis. The exact pathophysiology remains controversial but vasoconstriction, microarterial thrombosis, and ischemia might be implicated. Cocaineinduced pancreatitis is predominantly encountered in young, previously healthy patients. Therefore, a urine toxicology screen should be advocated in patients under 30 presenting with symptoms of pancreatitis. Future research should meticulously evaluate this association in large populationbased studies.

Funding The authors have no funding.

\section{Compliance with ethical standards}

Conflict of interest The authors declare that they have no conflict of interest.

Human rights All procedures followed have been performed in accordance with the ethical standards laid down in the 1964 Declaration of Helsinki and its later amendments.

Consent Informed consent was obtained from all patients for being included in the study.

\section{References}

1. Zimmerman JL. Cocaine intoxication. Crit Care Clin. 2012;28:517-26.

2. Inayat F, Bokhari SRA, Roberts L, et al. Cocaine-induced acute interstitial nephritis: a comparative review of 7 cases. J Investig Med High Impact Case Rep. 2020;8:2324709620932450.

3. Gourgoutis G, Das G. Gastrointestinal manifestations of cocaine addiction. Int J Clin Pharmacol Ther. 1994;32:136-41.

4. Muñiz AE, Evans T. Acute gastrointestinal manifestations associated with use of crack. Am J Emerg Med. 2001;19:61-3.

5. Bansal R, Sharma M, Aron J. Cocaine gut. ACG Case Rep J. 2019;6:e00041.

6. Sharma M, Rai P. Type 1 sphincter of Oddi dysfunction due to chronic opium addiction. Video J Encycl GI Endosc. 2013;2:599-601.

7. Alcazar-Guijo J, Jimenez-Saenz M, Gomez-Parra M, et al. Acute pancreatitis, incomplete thrombotic microangiopathy and cocaine abuse: an unusual pathogenic link. Intensive Care Med. 2005;31:1143.

8. Vazquez-Rodríguez S, Soto S, Fernandez E, et al. Cocaineinduced acute pancreatitis. Gastroenterol Hepatol. 2009;32:588.

9. Carlin N, Nguyen N, DePasquale JR. Multiple gastrointestinal complications of crack cocaine abuse. Case Rep Med. 2014;2014:512939.

10. Ogunbameru A, Jandali M, Issa A, et al. Acute pancreatitis as initial presentation of cocaine-induced vasculitis: a case report. JOP. 2015;16:192-4.

11. Chapela SP, de los Angeles PS, Ballestero FM. Pancreatitis induced by cocaine. Case Rep Gastroenterol. 2017;11:212-8.

12. Strzepka J, Tian F, Nassani N, et al. Cocaine-induced acute pancreatitis. ACG Case Rep J. 2020;7:e00487.

13. Umar M, Noor E, Ali U, et al. Cocaine-induced acute pancreatitis: a rare etiology. Cureus. 2020;12:e9029.

14. Testoni PA. Acute recurrent pancreatitis: etiopathogenesis, diagnosis and treatment. World J Gastroenterol. 2014;20:16891-901.

15. Bollen T. Acute pancreatitis: international classification and nomenclature. Clin Radiol. 2016;71:121-33.

16. Inayat F, Virk HU, Cheema AR, et al. Plasmablastic lymphoma mimicking acute pancreatitis. Case Rep Oncol Med. 2016;2016:9751736.

17. Substance Abuse and Mental Health Services Administration, Drug Abuse Warning Network, 2011: National Estimates of Drug-Related Emergency Department Visits. HHS Publication No. (SMA) 13-4760, DAWN Series D-39

18. Mederos MA, Reber HA, Girgis MD. Acute pancreatitis: a review. JAMA. 2021;325:382-90.

19. Moher D, Liberati A, Tetzlaff J, et al. Preferred reporting items for systematic reviews and meta-analyses: the PRISMA statement. PLoS Med. 2009;6:e1000097.

20. Banks PA, Bollen TL, Dervenis C, et al. Classification of acute pancreatitis-2012: revision of the Atlanta classification and definitions by international consensus. Gut. 2013;62:102e11.

21. Barkin JA, Simons-Linares RC, Nemeth Z, et al. Cocaineinduced acute pancreatitis: a systematic review: 56. Am J Gastroenterol. 2017;112:S27.

22. Inayat F, Zafar F, Baig AS, et al. Hypertriglyceridemic pancreatitis treated with insulin therapy: a comparative review of 34 cases. Cureus. 2018;10:e3501.

23. Chatila AT, Bilal M, Guturu P. Evaluation and management of acute pancreatitis. World J Clin Cases. 2019;7:1006-20.

24. Bernad M, Ibañez C, Medraño J, et al. Rhabdomyolysis in acute cocaine poisoning. Presentation of 2 cases. Paper presented at: Anales de medicina interna (Madrid, Spain: 1984); 1990. 
25. Lee KC, Ladizinski B, Federman DG. Complications associated with use of levamisole-contaminated cocaine: an emerging public health challenge. Mayo Clinic Proc. 2012;87:581-6.

26. Hackert T, Hartwig W, Fritz $\mathrm{S}$, et al. Ischemic acute pancreatitis: clinical features of 11 patients and review of the literature. Am J Surg. 2009;197:450-4.

27. Sakorafas GH, Tsiotos GG, Sarr MG. Ischemia/reperfusioninduced pancreatitis. Dig Surg. 2000;17:3-14.

28. Kanthasamy KA, Akshintala VS, Singh VK. Nutritional management of acute pancreatitis. Gastroenterol Clin N Am. 2021;50:141-50.

29. Inayat F, Zafar F, Riaz I, et al. Hypertriglyceridemic pancreatitis: is insulin monotherapy a feasible therapeutic option? Cureus. 2018; 10:e3461.

30. Ueki T, Kawamoto K, Otsuka Y, et al. Prevalence and clinicopathological features of autoimmune pancreatitis in Japanese patients with inflammatory bowel disease. Pancreas. 2015;44:434-40.

31. Shimosegawa T, Chari ST, Frulloni L, et al. International consensus diagnostic criteria for autoimmune pancreatitis: guidelines of the International Association of Pancreatology. Pancreas. 2011;40:352-8.

32. Inayat F, Virk HU, Yoon DJ, et al. Drug-induced pancreatitis: a rare manifestation of doxycycline administration. N Am J Med Sci. 2016;8:117-20.

33. Wolfe D, Kanji S, Yazdi F, et al. Drug induced pancreatitis: a systematic review of case reports to determine potential drug associations. PLoS ONE. 2020;15:e0231883.

34. Naranjo CA, Busto U, Sellers EM, et al. A method for estimating the probability of adverse drug reactions. Clin Pharmacol Ther. 1981;30:239-45.

Publisher's Note Springer Nature remains neutral with regard to jurisdictional claims in published maps and institutional affiliations. 